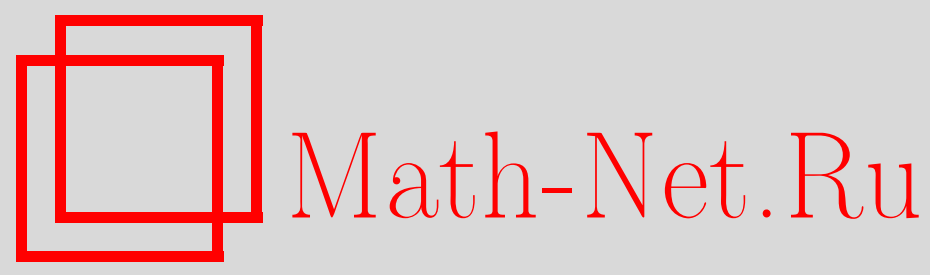

K. C. Рютин, Равномерная непрерывность обобщенных рациональных приближений, Матем. заметки, 2002, том 71, выпуск 2, 261-270

DOI: https://doi.org/10.4213/mzm345

Использование Общероссийского математического портала Math-Net.Ru подразумевает, что вы прочитали и согласны с пользовательским соглашением http://www.mathnet.ru/rus/agreement

Параметры загрузки:

IP : 3.80 .253 .173

26 апреля 2023 г., 18:05:05

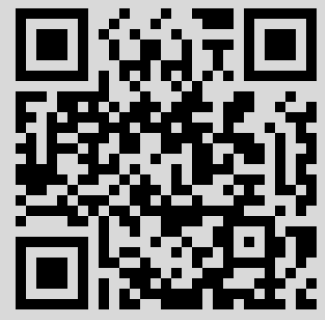




\title{
РАВНОМЕРНАЯ НЕПРЕРЫВНОСТЬ ОБОБЩЕННЫХ РАЦИОНАЛЬНЫХ ПРИБЛИЖЕНИЙ
}

\author{
К. С. Рютин
}

В работе показано, что не существует равномерно непрерывных мультипликативных $\varepsilon$-выборок из $C[0 ; 1]$ на множество обобщенных рациональных дробей достаточно общего вида при малых $\varepsilon$.

Библиография: 6 названий.

1. Введение. Работа посвящена доказательству отсутствия равномерно непрерывных $\varepsilon$-выборок на множество обобщенных рациональных дробей в $C[0 ; 1]$ при малых $\varepsilon$. Дадим несколько определений.

ОПредЕЛЕНИЕ. Пусть $X$ - метрическое пространство, $A \subset X, \varepsilon>0$. Для $x \in X$ положим $\rho(x, A)=\inf _{a \in A}\|x-a\|$. Отображение $\Phi: X \rightarrow A$ называется мультипликативной (соответственно аддитивной) $\varepsilon$-выборкой, если для любого $x \in X$ вьполнено неравенство

$$
\|\Phi(x)-x\| \leqslant(1+\varepsilon) \rho(x, A)
$$

(соответственно $\|\Phi(x)-x\| \leqslant \rho(x, A)+\varepsilon$ ); следовательно (в случае мультипликативной выборки), ограничение $\Phi$ на $A$ - тождественное отображение. Выборка назьвается непрерывной (липшичевой), если $\Phi$ непрерьвно (липшицево).

Для дальнейшего понадобится определить еще один важньй объект - обобщенные рациональные дроби. Всюду в дальнейшем $X$ - некоторое банахово пространство функций, определенных на отрезке $[0 ; 1]$; наиболее интересны случаи $X=L_{p}[0 ; 1]$ и $X=$ $C[0 ; 1]$.

ОПРЕДЕЛЕНИЕ. Пусть $V, W-$ подпространства $X$. Положим

$$
R_{V, W}=\left\{r=\frac{v}{w}: v \in V, w \in W, r \in X\right\} .
$$

Разумеется, мы предполагаем, что $R_{V, W} \neq \varnothing$ и $R_{V, W}$ - замкнутое подмножество $X$. Чтобы это было заведомо выполнено, мы в дальнейшем определим это множество более аккуратно. В случае, когда $V, W$ - подпространства многочленов степеней не выше $m$ и $n$ соответственно, вместо $R_{V, W}$ мы будем использовать стандартное обозначение $R_{m, n}$. Еще один важньй частный случай: $W=\langle 1\rangle$. Тогда $\operatorname{dim} W=1$ и $R_{V, W}=V-$

Работа выполнена при финансовой поддержке Российского фонда фундаментальных исследований, грант № 00-15-96109. 
подпространство в $X$. Здесь и всюду в дальнейшем под $\left\langle f_{1}, f_{2}, \ldots, f_{k}\right\rangle$, где $\left\{f_{j}\right\}_{j=1}^{k} \subset X$, a $k \in \mathbb{N}$, понимается линейная оболочка $f_{1}, f_{2}, \ldots, f_{k}$. У нас чаще всего $f_{j}$ будут выбираться линейно независимыми.

Первые результаты о существовании непрерывных $\varepsilon$-выборок на множества обобщенных рациональных дробей были получены Конягиньм, которьй доказал в [1] для любых $V, W$ и для любого $\varepsilon>0$ существование непрерьвной аддитивной $\varepsilon$-выборки из $C[0 ; 1]$ в $R_{V, W}$. Отсутствие непрерывной $\varepsilon$-выборки при малых $\varepsilon$ из пространства $L_{p}[0 ; 1], 1<p<\infty$, на множество $R_{m, n}, n>1$, доказано в работе Царькова [2]. Альбрехтом в работе [3] исследовался вопрос о существовании выборок на подпространства. Вопросами устойчивости непрерывных выборок занимался Маринов в [4]. Он, в частности, получил оценки устойчивости выборок через модуль выпуклости пространства. Конягину принадлежит первый отрицательньй результат о существовании равномерно непрерьвных операторов обобщенного рационального приближения. В работе [5] им анонсирована следующая

ТЕОремА А. При любом $\varepsilon \in(0 ; 2)$ не существует равномерно непрерывной $\varepsilon$-выборки из $C[0 ; 1]$ в $R_{0,1}$.

Доказательство этой теоремы содержится в работе автора [6]. В той же работе доказана следующая

ТЕОРемА В. Найдется $\varepsilon_{0}>0$ такое, что для любого $\varepsilon>\varepsilon_{0}$ cуществует лип-

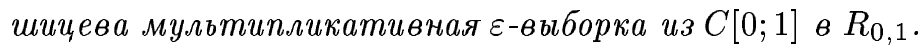

Для построения этой выборки был применен метод усреднения, использованньй в частной ситуации Альбрехтом в [3]. В этой работе мы получим обобщение теоремы Конягина [5].

Все рассматривавшиеся в работах [1], [2], [5], [6] множества $R_{V, W}$ состояли из вещественозначных обобщенных рациональных функций. Мы же рассматриваем как $\mathbb{R}$-, так и $\mathbb{C}$-значные рациональные дроби. Всюду в дальнейшем $X=C[0 ; 1]$ (пространство непрерьвных $\mathbb{R}$ - или же $\mathbb{C}$-значных функций с sup-нормой, каждый раз мы будем оговаривать особо, какое поле скаляров рассматриваем). Кроме того, мы рассматриваем только мультипликативные $\varepsilon$-выборки.

2. Формулировки теорем. В дальнейшем будем считать, что $\operatorname{dim} V, \operatorname{dim} W<\infty$; более того, $\operatorname{dim} V=1, \operatorname{dim} W=n \geqslant 2, V=\left\langle\varphi_{0}\right\rangle, W=\left\langle w_{1}, \ldots, w_{n}\right\rangle$. Кроме того, предполагаем, что $\left\{w_{j}\right\}_{j=1}^{n}-$ базис $W$. Наложим некоторые дополнительные ограничения на $V$ и $W$. Во-первых, всюду в дальнейшем считаем, что $X=C[0 ; 1]$ и $R_{V, W} \subset C[0 ; 1]$. Поэтому $\varphi_{0}, w_{j} \in C[0 ; 1]$. В зависимости от ситуации $V, W$ будут подпространствами в пространстве всех непрерьвных $\mathbb{R}-$ или же $\mathbb{C}$-значных функций. Теперь мы дадим аккуратное определение множества обобщенных рациональных дробей в $C[0 ; 1]$, которое годится и в действительном, и в комплексном случаях:

$$
R_{V, W}=\mathrm{Cl}\left\{r=\frac{v}{w}: v \in V, w \in W, w \neq 0 \text { на }[0 ; 1]\right\}
$$

где под $\mathrm{Cl} A$ понимается замыкание множества $A$.

Сначала наложим некоторые, впрочем довольно естественые, условия на $V, W$ в $\mathbb{R}$ случае; потом скажем, какие изменения надо внести, если функции $\mathbb{C}$-значные. 
УСловие 1. Для всех $\tau \in[0 ; 1]$ функиия $\varphi_{0}(\tau)>0$.

УСловие 2. Существует $f \in W$ такая, что $f(\tau)>0$ для всех $\tau \in[0 ; 1]$.

УСловиЕ 3. Множество нулей всякой функции $f \in W$ такой, что $f \not \equiv 0$, нигде не плотно на $[0 ; 1]$.

В $\mathbb{C}$-случае условия 1,2 надо сформулировать в виде: $\varphi_{0}(\tau) \neq 0$ для всех $\tau \in[0 ; 1]$ и сушествует $f \in W$ такая, что $f(\tau) \neq 0$ для всех $\tau \in[0 ; 1]$. Условие 3 переносится на $\mathbb{C}$-случай дословно. Из условия 1 следует (в $\mathbb{R}$-случае) существование $\alpha>0$ такого, что $\varphi_{0}(\tau)>\alpha$ для всех $\tau \in[0 ; 1]$. Из-за нетривиальности $R_{V, W}$ приходим к необходимости условия 2 .

Условию 3 удовлетворяют аналитические функции и, в частности, многочлены. Договоримся писать $R_{\mathbb{R}}$ или $R_{\mathbb{C}}$ в зависимости от того, какие дроби (вещественные или же комплексные) мы рассматриваем.

ЗАМЕчАнИЕ. Если вьполнены условия 1-3 на подпространства $V, W$, то

$$
\left\{r=\frac{v}{w}: v \in V, w \in W, w \neq 0 \text { на }[0 ; 1]\right\}
$$

- замкнутое подмножество $C[0 ; 1]$ и, следовательно, совпадает с $R_{V, W}$.

Убедимся в справедливости этого замечания. Пусть $r^{k}=\varphi_{0} / w^{k} \rightarrow r^{0} \in C[0 ; 1]$ при $k \rightarrow \infty$, где $w^{k} \in W$ - знаменатель $k$-й дроби. Достаточно показать, что $w^{k}$ стремится к некоторому элементу $w^{0} \in W$, так как из этого следует, что $w^{0}$ не обращается в нуль на [0; 1$]$ (в противном случае $r^{0} \notin C[0 ; 1]$ ). Предположим, что $\left\{w^{k}\right\}_{k=1}^{k=\infty}$ - неограниченная последовательность в $W$, в противном случае из нее можно выбрать сходящуюся подпоследовательность. Покажем, что $r^{0} \equiv 0$. Рассмотрим $\widetilde{w}^{k} \in W$ такие, что $w^{k}=\left\|w^{k}\right\| \widetilde{w}^{k}$. Так как для любого $k\left\|\widetilde{w}^{k}\right\|=1$, то из последовательности $\left\{\widetilde{w}^{k}\right\}$ можно извлечь сходящуюся подпоследовательность. Пусть $\widetilde{w}^{k} \rightarrow w^{0}$. Заметим, что $\left\|w^{0}\right\|=1$ и, следовательно, множество нулей функции $w^{0}$ нигде не плотно на отрезке $[0 ; 1]$ в силу условия 3 на $W$. Следовательно, из последовательности $w^{k}$ можно извлечь подпоследовательность, которая на всюду плотном подмножестве отрезка $[0 ; 1]$ стремится к $\infty$. Тем самым, $r^{0}=0$ на всюду плотном множестве, а следовательно, $r^{0} \equiv 0$.

Этим замечанием мы будем пользоваться при всех дальнейших рассмотрениях.

Теорема 1. Пусть $V, W$ удовлетворяют условиям 1-3. Тогда для любого $\varepsilon \in$ $(0 ; 2)$ не существует равномерно непрерывной мультипликативной $\varepsilon$-выборки из шара в $C[0 ; 1]$ на $R_{\mathbb{R}}=R_{V, W}$.

В $\mathbb{C}$-случае ограничение на $\varepsilon$ получается хуже.

ТЕОРема 2. Пусть $V, W$ удовлетворяют условиям 1-3. Тогда для любого $\varepsilon \in$

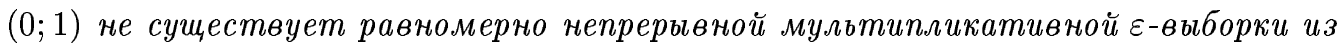
шара в $C[0 ; 1]$ на $R_{\mathbb{C}}=R_{V, W}$.

Наложенные в теореме 1 условия на подпространства $V, W$ не являются необходимыми. Автору известны некоторые случаи, когда теорема 1 справедлива и при этом $\varphi_{0}$ имеет нули на отрезке [0; 1 . Получены и некоторые результаты, аналогичные теореме 1 , при $\operatorname{dim} V>1$. 
3. Вспомогательные утверждения. Доказательство в случае вещественного и комплексногополя скаляров идет по единой схеме, поэтому будет вестись одновременно. Если какие-то детали доказательства будут отличаться, мы будем всякий раз оговаривать, как надо действовать в $\mathbb{R}$ - или же $\mathbb{C}$-случае. Сформулируем лемму, справедливую в обоих случаях.

Лемма 1. Пусть $W$ - подпространство в $C[0 ; 1]$, для которого выполнено условие $3 u \operatorname{dim} W=n$. Предположим дополнительно, что найдется точка $\tau_{0} \in[0 ; 1)$, в которой $f\left(\tau_{0}\right)=0$ для всех $f \in W$. Тогда для любих $a>0, \varepsilon>0$ найдется $\delta>0$ такое, что для всякой функиии $f \in W$ такой, что $\|f\|_{C\left[\tau_{0} ; \tau_{0}+\varepsilon\right]} \leqslant 1$, выполнено неравенство $\|f\|_{C\left[\tau_{0} ; \tau_{0}+\delta\right]} \leqslant a$.

ДокАЗАтЕльство. Доказьвать лемму будем сразу в $\mathbb{R}$ - и $\mathbb{C}$-случаях. Обозначим через $\mathbb{K}$ рассматриваемое поле скаляров. Пусть $f=\sum_{j=1}^{n} a_{j} w_{j}$, где $\left\{w_{j}\right\}$-стандартный базис $W$, а $a_{j} \in \mathbb{K}$. В силу условий леммы $w_{j}\left(\tau_{0}\right)=0$ для всех $j$. Будем обозначать той же буквой $W$ подпространство в $C\left[\tau_{0} ; \tau_{0}+\varepsilon\right]$, порожденное ограничениями функций из $W$ на отрезок $\left[\tau_{0} ; \tau_{0}+\varepsilon\right]$.

Введем на $W$ две нормы:

$$
\|f\|_{1}=\sum_{j=1}^{n}\left|a_{j}\right|, \quad\|f\|_{2}=\|f\|_{C\left[\tau_{0} ; \tau_{0}+\varepsilon\right]} .
$$

Заметим, что $\|f\|_{2}$ является нормой по условию 3 на подпространство $W$. Подпространство $W$ конечномерное, поэтому на нем все нормы эквивалентны. Следовательно, найдется $C>0$ такое, что из $\|f\|_{2} \leqslant 1$ следует

$$
\|f\|_{1}=\sum_{j=1}^{n}\left|a_{j}\right| \leqslant C .
$$

Теперь выберем $\delta>0$ со свойством $\omega\left(w_{j}, \delta\right) \leqslant a / C$ для всех $j$, где через $\omega(g, \delta)$ обозначен модуль непрерывности функции $g$. Для произвольного числа $\tau \in(0 ; \delta]$ имеем

$$
\left|f\left(\tau_{0}+\tau\right)\right|=\left|f\left(\tau_{0}+\tau\right)-f\left(\tau_{0}\right)\right| \leqslant \sum_{j=1}^{n}\left|a_{j}\right| \max _{j} \omega\left(w_{j}, \delta\right) \leqslant a .
$$

Заметим, что если зафиксировать $a$, то в лемме фактически построена функция $\delta=$ $\delta(\varepsilon)$, которую можно считать монотонно возрастающей.

Теперь приступим к построению удобного для доказательства теоремы базиса в подпространстве $W$.

Лемма 2. А) $\mathbb{C}$-случай. Пусть $W-$ подпространство в $C[0 ; 1] u \operatorname{dim} W \geqslant 2$. Предположим дополнительно, что найдется $f \in W$, которая не обращается в 0 на $[0 ; 1]$. Тогда для любого числа $A \in(1 ; 2)$ существуют линейно независимые функиии $w_{1}, w_{2} \in W$ и число $\tau_{0} \in[0 ; 1]$, для которых будут выполнены следующие условия:

1) $w_{2}\left(\tau_{0}\right)=0, w_{1}+M w_{2} \neq 0$ на $[0 ; 1]$ для всех $M>0$ и, более того,

$$
\left\|\frac{1}{w_{1}+M w_{2}}\right\| \leqslant 1 \quad \text { для всех } M>0 ;
$$

2) $\left\|\frac{1}{w_{1}}\right\|=1,\left|w_{1}\left(\tau_{0}\right)\right|<A u w_{1} \neq 0$ на $[0 ; 1]$. 
В) $\mathbb{R}$-случай. Пусть $W-$ подпространство в $C[0 ; 1] u \operatorname{dim} W \geqslant 2$. Предположим дополнительно, что найдется положительная $f \in W$. Тогда для любого числа $A \in(1 ; 2)$ существуют линейно независимые функиии $w_{1}, w_{2} \in W$ и число $\tau_{0} \in[0 ; 1]$, для которых будут выполнены следующие условия:

1) $w_{2}\left(\tau_{0}\right)=0, w_{2} \geqslant 0 \mu a[0 ; 1]$

2) $\left\|\frac{1}{w_{1}}\right\|=1, w_{1}\left(\tau_{0}\right)<A$ и $w_{1}>0 н а[0 ; 1]$.

Заметим, что для рассматриваемого в теореме 1 подпространства $W$ вьполнены все условия леммы 2 .

ДокАЗАТЕльСтво. Будем вести доказательство случая А), указьвая, какие изменения надо вносить в доказательство, чтобы получить случай В). По условию леммы имеется функция $f \in W$, которая нигде не обращается в 0 на $[0 ; 1]$. Кроме того, найдется линейно независимая с $f$ функция $g \in W$.

Обозначим $\Omega_{g}=\{z \in \mathbb{C}: \exists \tau \in[0 ; 1](z f+g)(\tau)=0\}$. Заметим, что $\Omega_{g}$ - компактное подмножество $\mathbb{C}$. Пусть точка $z_{0}=x_{0}+i y_{0} \in \Omega_{g}$ такая, что

$$
x_{0}=\max _{z \in \Omega_{g}}\{\operatorname{Re} z\}, \quad f_{2}=z_{0} f+g .
$$

Из линейной независимости $f$ и $g$ следует $f_{2} \not \equiv 0$. Построим аналогичную функцию $f_{2}$ в случае В). По условию В) имеется функция $f \in W$ такая, что $f>0$ на $[0 ; 1]$. Кроме того, найдется линейно независимая с $f$ функция $g \in W$, которая может, вообще говоря, принимать как положительные, так и отрицательные значения на отрезке [0;1]. Положим

$$
\lambda_{0}=\inf _{\lambda}\{\lambda: \lambda f+g \geqslant 0 \text { на }[0 ; 1]\}, \quad f_{2}=\lambda_{0} f+g .
$$

Из линейной независимости $f$ и $g$ следует $f_{2} \not \equiv 0$. Далее доказательство идет сходным образом в обоих случаях А) и В). Установим одно свойство функций $f, f_{2}$, справедливое в обоих случаях. Для любой точки $\tau \in[0 ; 1]$ и числа $s>0$ справедливо неравенство

$$
\left|\left(s f+f_{2}\right)(\tau)\right| \geqslant \max \left\{s|f(\tau)|,\left|f_{2}(\tau)\right|\right\} .
$$

В вешественном случае неравенство (1) очевидно (обе функции $f$ и $f_{2}$ неотрицательны) и, более того, можно убрать модули. Установим неравенство (1) в случае А). По нашему выбору $f, f_{2}$ для любого $z \in \mathbb{C}$ такого, что $\operatorname{Re} z>0$, выполнено следующее: $f_{2}+z f$ не обрашается в 0 на $[0 ; 1]$. Из этого следует, что для любого $\tau \in[0 ; 1]$ такого, что $f_{2}(\tau) \neq 0$, выполнено

$$
\left|\arg f_{2}(\tau)-\arg f(\tau)\right| \leqslant \frac{\pi}{2} .
$$

Тем самым, требуемое неравенство (1) установлено.

Пусть

$$
T=\left\{\tau \in[0 ; 1]: f_{2}(\tau)=0\right\}
$$

- множество нулей $f_{2}$. Тогда $T$ - компактное собственное подмножество отрезка $[0 ; 1]$. Следовательно, найдется $\tau_{0} \in T$ такое, что $f\left(\tau_{0}\right)=\min _{\tau \in T}|f(\tau)|$. Построение функции $w_{1}$ проводится единьм образом в обоих случаях А), В). Рассмотрим семейство функций из $C[0 ; 1]: f_{s}=s f+f_{2}$, где $s \geqslant 0$ - "мальй параметр". По нашим построениям $f_{s} \neq 0$ 
на $[0 ; 1]$ для всех $s>0$. Для доказательства леммы достаточно показать, что найдется $s_{0}>0$, для которого будет выполнено условие

$$
\frac{\left|f_{s_{0}}\left(\tau_{0}\right)\right|}{\min _{\tau \in[0 ; 1]}\left|f_{s_{0}}(\tau)\right|}<A
$$

Заметим, что $f_{s}\left(\tau_{0}\right)=s f\left(\tau_{0}\right)$ для всех $s \geqslant 0$.

Рассмотрим покрытиемножества $T$ открытьми интервалами $U_{j}$ такими, что для любого $j$ и для любых $\tau_{1}, \tau_{2} \in U_{j}$ выполнено

$$
\left|f\left(\tau_{1}\right)-f\left(\tau_{2}\right)\right| \leqslant \frac{A-1}{A}\left|f\left(\tau_{0}\right)\right|
$$

Благодаря компактности $T$ такой набор множеств $U_{j}$ можно считать конечным. Рассмотрим $D=[0 ; 1] \backslash\left(\bigcup_{j} U_{j}\right)$. Разумеется, $D$ - компактноеподмножество [0; 1$]$. Поэтому существует

$$
\min _{\tau \in D}\left|f_{2}(\tau)\right|=h>0 .
$$

Теперь займемся выбором необходимого нам значения $s_{0}$. Если $\tau \in \bigcup_{j} U_{j}$, то по неравенству (1)

$$
\left|f_{s}(\tau)\right| \geqslant s|f(\tau)| \geqslant s\left(|f(z)|-\frac{A-1}{A}\left|f\left(\tau_{0}\right)\right|\right) \geqslant s \frac{\left|f\left(\tau_{0}\right)\right|}{A}
$$

мы выбрали некоторьй $z \in T$ таким образом, что $z, \tau$ принадлежат одному $U_{j}$. Если $\tau \in D$, то

$$
\left|f_{s}(\tau)\right| \geqslant\left|f_{2}(\tau)\right| \geqslant h>s \frac{\left|f\left(\tau_{0}\right)\right|}{A} .
$$

Самое левое неравенство вьполнено при $s<h A /\left|f\left(\tau_{0}\right)\right|$. Выберем какое-нибудь число $s_{0}$, удовлетворяющее этому неравенству, и отвечающую ему функцию $f_{s_{0}}$.

Нормируем $f_{s_{0}}$ таким образом, что для функции $w_{1}=\alpha f_{s_{0}}, \alpha>0$, вьполнено

$$
\left\|\frac{1}{w_{1}}\right\|=1
$$

Обозначим $w_{2}:=f_{2}$. Из построения вытекает, что $w_{1}+M w_{2} \neq 0$ на $[0 ; 1]$ для всех $M>0$. Более того, из неравенства (1) следует, что

$$
\left\|\frac{1}{w_{1}+M w_{2}}\right\| \leqslant 1
$$

для всех $M>0$. Итак, требуемые $w_{1}, w_{2}$ построены.

Дополним построенные в лемме $2 w_{1}, w_{2}$ до базиса $W$. Можно считать $w_{j}\left(\tau_{0}\right)=0$ для всех $j \geqslant 2$. Теперь можно перейти собственно к доказательству теоремы 1 .

4. Доказательство теорем 1 и 2. Доказьваются обе теоремы одним и тем же рассуждением, только в одном месте при доказательстве можно сделать в вещественном случае более точную оценку. Доказательству теоремы предпошлем 
ЗАмечАниЕ 1 . Достаточно доказьвать теорему в предположении $\varphi_{0} \equiv 1$, ибо в силу однородности $R_{V, W}$ можно от пары $(V, W)$ перейти к $(\widetilde{V}, \widetilde{W})$, которая задает такое же $R_{V, W}$. А именно, $\widetilde{V}=\langle 1\rangle, \widetilde{W}=\left\langle w_{1} / \varphi_{0}, \ldots, w_{n} / \varphi_{0}\right\rangle$. Заметим, что для $\widetilde{W}$, по-прежнему, вьполнены условия 2 и 3.

Доказывать теорему будем методом от противного. Пусть $\Phi$ - равномерно непрерывная мультипликативная $\varepsilon$-выборка из единичного шара в $C[0 ; 1]$ на $R_{V, W}, 0<\varepsilon<2$. Через $\omega(\delta)$ обозначим модуль непрерывности отображения $\Phi$. Для сокращения записи обозначим $R=R_{V, W}$. Благодаря замечанию 1 можно считать, что $V=\langle 1\rangle$. Обозначим $x_{0}=1 / w_{1}, x_{1}=1 /\left(w_{1}+M w_{2}\right), M>1$ - число. Мы используем построенньй вьше базис $\left\{w_{j}\right\}$. В дальнейшем мы устремим $M$ к $+\infty$. Рассмотрим отрезок

$$
x_{\lambda}=(1-\lambda) x_{0}+\lambda x_{1}, \quad \lambda \in[0 ; 1],
$$

который лежит в единичном шаре пространства $C[0 ; 1]$. Введем следующие обозначения:

$$
\Phi\left(x_{\lambda}\right)=\frac{a_{\lambda}}{w_{1}+p_{\lambda}}, \quad p_{\lambda} \in\left\langle w_{2}, \ldots, w_{n}\right\rangle .
$$

Заметим, что $p_{\lambda}$ однозначно определяется по $\Phi, x_{\lambda}$ и $p_{\lambda}\left(\tau_{0}\right)=0$ для всех $\lambda \in[0 ; 1]$. Оценим

$$
\left\|\Phi\left(x_{\lambda}\right)-\Phi\left(x_{\mu}\right)\right\| \leqslant \omega\left(\left\|x_{\lambda}-x_{\mu}\right\|\right)=\omega\left(|\lambda-\mu| \cdot\left\|x_{0}-x_{1}\right\|\right) .
$$

Применив неравенство $\omega(k \delta) \leqslant k \omega(\delta)$, справедливое для любого числа $k \in \mathbb{N}$ и любого модуля непрерьвности, получим

$$
\left\|\Phi\left(x_{\lambda}\right)-\Phi\left(x_{\mu}\right)\right\| \leqslant \omega(2|\lambda-\mu|) \leqslant 2 \omega(|\lambda-\mu|) .
$$

Следовательно,

$$
\frac{\left|a_{\lambda}-a_{\mu}\right|}{\left|w_{1}\left(\tau_{0}\right)\right|}=\left|\Phi\left(x_{\lambda}\right)\left(\tau_{0}\right)-\Phi\left(x_{\mu}\right)\left(\tau_{0}\right)\right| \leqslant\left\|\Phi\left(x_{\lambda}\right)-\Phi\left(x_{\mu}\right)\right\| \leqslant 2 \omega(|\lambda-\mu|) .
$$

Итак,

$$
\left|a_{\lambda}-a_{\mu}\right| \leqslant L \omega(|\lambda-\mu|),
$$

где $L>0$ не зависит от выбора $x_{0}, x_{1}, M$. Покажем, что для всех $\lambda \in[0 ; 1]$

$$
\rho\left(x_{\lambda}, R\right) \leqslant \frac{1}{2} .
$$

В самом деле, $\lambda x_{1},(1-\lambda) x_{0} \in R$, поэтому

$$
\rho\left(x_{\lambda}, R\right) \leqslant \min \left\{\lambda\left\|x_{1}\right\|,(1-\lambda)\left\|x_{0}\right\|\right\} \leqslant \frac{1}{2} .
$$

В вещественном случае (теорема 1) эту оценку можно усилить. А именно, вьполнено неравенство

$$
\rho\left(x_{\lambda}, R\right) \leqslant \frac{1}{3} .
$$


Докажем его. Возможны два случая: $\lambda \in[0 ; 1 / 3] \cup[2 / 3 ; 1]$ и $\lambda \in[1 / 3 ; 2 / 3]$. В первом из них требуемое неравенство очевидно. В самом деле, $\lambda x_{1},(1-\lambda) x_{0} \in R$, поэтому

$$
\rho\left(x_{\lambda}, R\right) \leqslant \min \left\{\lambda\left\|x_{1}\right\|,(1-\lambda)\left\|x_{0}\right\|\right\} \leqslant \frac{1}{3} .
$$

Во втором можно приблизить $x_{\lambda}$ функцией

$$
\frac{2}{3} x_{0}=\frac{2}{3 w_{1}} \in R,
$$

и требуемое неравенство получится. А именно,

$$
\rho\left(x_{\lambda}, R\right) \leqslant\left\|x_{\lambda}-\frac{2}{3 w_{1}}\right\| \leqslant \frac{1}{3} .
$$

Докажем последнее неравенство. Имеем

$$
-\frac{1}{3} x_{0} \leqslant(1-\lambda) x_{0}-\frac{2}{3} x_{0} \leqslant(1-\lambda) x_{0}+\lambda x_{1}-\frac{2}{3} x_{0} \leqslant(1-\lambda) x_{0}+\lambda x_{0}-\frac{2}{3} x_{0}=\frac{1}{3} x_{0} .
$$

При доказательстве теоремы 2 , используя определение мультипликативной $\varepsilon$-выборки и применяя неравенство (2), имеем

$$
\left\|\Phi\left(x_{\lambda}\right)-x_{\lambda}\right\| \leqslant \frac{1}{2}(1+\varepsilon) .
$$

Используя явньй вид $x_{\lambda}$, получаем

$$
\left|\frac{1}{w_{1}\left(\tau_{0}\right)}-\frac{a_{\lambda}}{w_{1}\left(\tau_{0}\right)}\right| \leqslant \frac{1}{2}(1+\varepsilon),
$$

откуда следует неравенство

$$
\left|a_{\lambda}\right| \geqslant 1-\frac{1}{2}\left|w_{1}\left(\tau_{0}\right)\right|(1+\varepsilon)
$$

Поэтому при $\varepsilon<1$ выполнено $(1+\varepsilon) / 2<1$, и согласно утверждению А) леммы 2 можно выбрать $w_{1}$ так, что

$$
\frac{1}{2}\left|w_{1}\left(\tau_{0}\right)\right|(1+\varepsilon)<1 .
$$

Итак, при всех $\lambda \in[0 ; 1]$ выполнено

$$
\left|a_{\lambda}\right| \geqslant \beta_{0},
$$

где $\beta_{0}>0$ и не зависит от выбора $M$.

Аналогичным образом при доказательстве теоремы 1 , используя определение мультипликативной $\varepsilon$-выборки, неравенство (3) и явньй вид $x_{\lambda}$, получим, что при любом $\varepsilon<2$ согласно утверждению В) леммы 2 можно выбрать $w_{1}$ так, что

$$
\frac{1}{3} w_{1}\left(\tau_{0}\right)(1+\varepsilon)<1
$$


И, следовательно, при всех $\lambda \in[0 ; 1]$ выполнено

$$
a_{\lambda} \geqslant \beta_{0}
$$

где $\beta_{0}>0$ не зависит от выбора $M$.

В дальнейшем доказательство теорем 1 и 2 проходит одинаково. Из наших рассмотрений следует существование константы $C>0$, не зависящей от параметра $M$, такой, что для любых $\lambda, \mu \in[0 ; 1]$

$$
\left\|\frac{1}{w_{1}+p_{\lambda}}-\frac{1}{w_{1}+p_{\mu}}\right\| \leqslant C \omega(|\lambda-\mu|) .
$$

В самом деле,

$$
\begin{aligned}
\left|a_{\lambda}\right| \cdot\left\|\frac{1}{w_{1}+p_{\lambda}}-\frac{1}{w_{1}+p_{\mu}}\right\|-\left|a_{\lambda}-a_{\mu}\right| \cdot\left\|\frac{1}{w_{1}+p_{\mu}}\right\| & \leqslant\left\|\frac{a_{\lambda}}{w_{1}+p_{\lambda}}-\frac{a_{\mu}}{w_{1}+p_{\mu}}\right\| \\
& \leqslant 2 \omega(|\lambda-\mu|) .
\end{aligned}
$$

Введем важную величину

$$
t_{\lambda}=\inf \left\{t>0:\left\|p_{\lambda}\right\|_{C\left[\tau_{0} ; \tau_{0}+t\right]}=1,\left|p_{\lambda}(s)\right|<1 \quad \forall s: 0 \leqslant s<t\right\},
$$

если этот inf существует и принадлежит интервалу $\left(0 ; 1-\tau_{0}\right]$, в противном случае $t_{\lambda}:=1-\tau_{0}$. Разумеется, мы предполагаем (и это не ограничивает обшности), что $\tau_{0} \neq 1$. Вспомним, что $p_{\lambda}\left(\tau_{0}\right)=0$, поэтому если требуемый inf существует, то он не равен нулю.

Пусть $t_{\lambda}>t_{\mu}$. Тогда

$$
\begin{aligned}
\left\|\frac{1}{w_{1}+p_{\lambda}}-\frac{1}{w_{1}+p_{\mu}}\right\| & \geqslant\left\|\frac{p_{\lambda}-p_{\mu}}{\left(w_{1}+p_{\lambda}\right)\left(w_{1}+p_{\mu}\right)}\right\|_{C\left[\tau_{0} ; \tau_{0}+t_{\mu}\right]} \\
& \geqslant \frac{\left\|p_{\lambda}-p_{\mu}\right\|_{C\left[\tau_{0} ; \tau_{0}+t_{\mu}\right]}}{\left(n_{1}+1\right)^{2}} \geqslant \gamma\left(1-\left\|p_{\lambda}\right\|_{C\left[\tau_{0} ; \tau_{0}+t_{\mu}\right]}\right),
\end{aligned}
$$

где $n_{1}=\left\|w_{1}\right\|_{C[0 ; 1]}$, а $\gamma>0$ не зависит от выбора $M$. Итак, мы получили, что при $t_{\lambda}>t_{\mu}$ вьполнено неравенство

$$
\left\|p_{\lambda}\right\|_{C\left[\tau_{0} ; \tau_{0}+t_{\mu}\right]} \geqslant 1-A \omega(|\lambda-\mu|),
$$

где $A>0$ не зависит от $M, \lambda, \mu$. Вспомним, что в лемме 1 фактически построена монотонно возрастающая функция $\delta=\delta(\varepsilon)$ (при фиксированном $a>0$ ). Мы применяем эту лемму к $\left\langle w_{2}, \ldots, w_{n}\right\rangle$. Рассмотрим равномерное разбиение отрезка $[0 ; 1]$ на $N$ частей

$$
\lambda_{0}=0<\lambda_{1}=\frac{1}{N}<\cdots<\lambda_{N}=1 .
$$

Число $N$ мы выберем достаточно большим для того, чтобы для всех $0 \leqslant j<N$ было выполнено $a:=1-A \omega\left(\left|\lambda_{j}-\lambda_{j+1}\right|\right)>0$. 
Чтобы не использовать двойные индексы, будем писать $p_{j}$ вместо $p_{\lambda_{j}}$ и $t_{j}$ вместо $t_{\lambda_{j}}$. Заметим, что $t_{0}=1-\tau_{0}$ и $t_{N} \rightarrow 0$ при $M \rightarrow \infty$. В самом деле, так как множество нулей $w_{2}$ нигде не плотно на $[0 ; 1]$, на любом интервале $(0 ; d)$ найдется точка $\widehat{\tau}$ такая, что $\left|w_{2}\left(\tau_{0}+\widehat{\tau}\right)\right|>0$, поэтому из $M>1 /\left|w_{2}\left(\tau_{0}+\widehat{\tau}\right)\right|$ следует $t_{N} \in(0 ; \widehat{\tau})$. Используя лемму 1 (монотонность функции $\delta(\varepsilon)$, построенной в этой лемме), получим

$$
t_{1}>\delta\left(t_{0}\right), \quad t_{2}>\delta\left(t_{1}\right)>\delta \circ \delta\left(t_{0}\right)
$$

Учитьвая, что $t_{0}=1-\tau_{0}$, имеем

$$
t_{N}>\delta \circ \delta \circ \cdots \circ \delta\left(1-\tau_{0}\right)>0 .
$$

В последнем неравенстве правая часть не зависит от $M$, а левая стремится к 0 при $M \rightarrow \infty$. Полученное противоречие доказьвает теорему.

Автор благодарит С. В. Конягина за постановку задач и общее руководство работой; В.М. Тихомирова и И.Г. Царькова за помощь в редактировании и большое количество замечаний, которые способствовали значительному улучшению текста.

\section{СПИСОК ЦИТИРОВАННОЙ ЛИТЕРАТУРЫ}

[1] Конягин С. В. О непрерьвности операторов обобщенного рационального приближения // Матем. заметки. 1988. Т. 44. №3. С. 404.

[2] Царьков И. Г. Свойства множеств, обладающих непрерывной выборкой из оператора $P^{\delta}$ // Матем. заметки. 1990. Т. 48. № 4. С. 122-131.

[3] Альбрехт П. В. Порядки модулей непрерьвности операторов почти наилучшего приближения // Матем. сб. 1994. Т. 185. № 9. С. 3-28.

[4] Маринов А. В. Оценки устойчивости непрерывной селекции для метрической почти-проекции // Матем. заметки. 1994. Т. 55. № 4. С. 47-53.

[5] Конягин С. В. О равномерной непрерьвности операторов рационального приближения. Теория приближений и задачи вычислительной математики. Т. 108. Днепропетровск, 1993.

[6] Рютин К. С. Липшицевость ретракций и оператор обобщенного рационального приближения // Фундамент. и прикл. матем. 2000. Т. 6. № 4. С. 1205-1220.

Московский государственный университет им. М.В. Ломоносова E-mail: rutin@mccme.ru 